\title{
ON INCOMPARABILITY OF BANACH SPACES
}

\author{
MANUEL GONZÁLEZ \\ Departamento de Matemáticas, Universidad de Cantabria \\ 39071 Santander, Spain \\ ANTONIO MARTINÓN \\ Departamento de Análisis Matemático, Universidad de La Laguna \\ 38271 La Laguna (Tenerife), Spain
}

\begin{abstract}
Several concepts of incomparability of Banach spaces have been considered in the literature, which allow one to describe some of the properties of the product of two Banach spaces as a juxtaposition of the corresponding properties of the factors. In this paper we study the relations between these concepts of incomparability, survey the main results and applications, and state some open problems.
\end{abstract}

Introduction. One topic in which the concepts and results of the theory of Fredholm operators have been fruitfully applied in Banach space theory is the study of incomparability relations for Banach spaces. In [40], Rosenthal says that two Banach spaces $X$ and $Y$ are totally incomparable if no infinite-dimensional (closed) subspace of $X$ is isomorphic to a subspace of $Y$. Previously, Gurariî [21] called such a pair of spaces essentially nonisomorphic. Rosenthal gave a characterization of this concept that has been useful in many situations [2], [22], [24], [41]. Edelstein and Wojtaszczyk [13] proved that given two totally incomparable Banach spaces $X$ and $Y$, any complemented subspace of the direct sum $X \oplus Y$ is isomorphic to the product of a complemented subspace of $X$ and a complemented subspace of $Y$. Later, Wojtaszczyk [44] extended the result to the case where all operators from $X$ into $Y$ are strictly singular. These results were included in [28, 2.c]. Later on, other concepts of incomparability were introduced and applied.

1991 Mathematics Subject Classification: Primary 46B20.

The paper is in final form and no version of it will be published elsewhere.

Research supported in part by DGICYT Grant PB91-0307 (Spain). 
In this paper we survey the main concepts of incomparability of Banach spaces and their applications. In the first section we give the definitions of the incomparability concepts and show the relations between them. We distinguish two types of incomparability: structural incomparability and operational incomparability. We emphasize the importance of essential incomparability because it admits an operational definition and some structural characterizations, and it is one of the widest incomparability concepts.

In the second section we summarize the applications that incomparability have found in several topics of the theory of Banach spaces: complemented subspaces, unconditional bases of finite products, structure of tensor products, spaces whose group of invertible operators is not connected, construction of space ideals with the three-space property, and description of classes of operators defined in terms of operational quantities.

Notations. The letters $X$ and $Y$ will denote real or complex Banach spaces; by a subspace we shall always mean a closed subspace. Also, $L(X, Y)$ will be the space of all (linear continuous) operators from $X$ into $Y$. An operator will be called an injection if it is one-to-one and has closed range (i.e., if it is an isomorphism into), and it will be called a surjection if it is surjective. We will denote by $J_{M}$ the canonical injection of a subspace $M$ of $X$ into $X$, and by $Q_{M}$ the quotient map from $X$ onto $X / M$.

1. Incomparability concepts. In this section we define several notions of incomparability. We distinguish two types: structural incomparability, defined in terms of the structure of the spaces, and operational incomparability, defined using classes of operators. We give the relations between the different concepts of incomparability and study their duality properties.

Structural incomparability. Three concepts of structural incomparability are considered: subspace incomparability, quotient incomparability and projection incomparability. The first one was introduced by Gurariu [21], in a paper published in Russian, who uses the term essentially nonisomorphic spaces, and Rosenthal [40], who uses the term totally incomparable spaces, but we prefer subspace incomparable spaces.

1.1. Definition [40]. The Banach spaces $X$ and $Y$ are said to be subspace incomparable if there is no infinite-dimensional subspace of $X$ isomorphic to a subspace of $Y$.

The following theorem gives a necessary and sufficient condition for two Banach spaces to be subspace incomparable. The necessary condition was obtained earlier in [21].

1.2. TheOREM [40, Theorem 2]. The Banach spaces $X$ and $Y$ are subspace incomparable if and only if for any Banach space $Z$, and subspaces $X_{0}$ and $Y_{0}$ of $Z$ isomorphic to $X$ and $Y$ respectively, the sum $X_{0}+Y_{0}$ is closed. 
This result has been applied in [2], [3], [22], [24] and [41] in order to show that certain sums of subspaces are closed.

1.3. R e mark. In 1973, Diestel and Lohman [9, Theorem 4] extended Definition 1.1 to Hausdorff locally convex spaces, and proved that the direct implication in Theorem 1.2 is valid in this situation. In the same year, Lohman [30] gave an example of a nonmetrizable Hausdorff locally convex space $Z$ such that $Z=$ $X+Y, X \cap Y=\{0\}$, with $X$ and $Y$ Banach spaces. Drewnowski [12, Theorem 4.1] proved a similar result for Fréchet spaces; in the same direction, see the paper of Kalton [23].

In 1986, Díaz [8] uses the fact that the Banach sequence spaces $\ell_{p}$ and $\ell_{q}$ are subspace incomparable for $p \neq q$ to show an example of a Fréchet non-Montel space without infinite-dimensional normable subspaces.

A dual incomparability concept was introduced in [18], using quotients instead of subspaces in Definition 1.1. In that paper the term totally coincomparable is used, but we prefer quotient incomparable.

1.4. Definition [18, Definition]. The Banach spaces $X$ and $Y$ are said to be quotient incomparable if there is no infinite-dimensional quotient of $X$ isomorphic to a quotient of $Y$.

In [18], a simpler proof of Theorem 1.2 and an analogous result for quotient incomparable spaces are given.

1.5. Theorem [18, Theorem]. The Banach spaces $X$ and $Y$ are quotient incomparable if and only if for any Banach space $Z$, and subspaces $X_{0}$ and $Y_{0}$ of $Z$ such that $Z / X_{0}$ and $Z / Y_{0}$ are isomorphic to $X$ and $Y$ respectively, the sum $X_{0}+Y_{0}$ is closed.

The above characterizations of subspace incomparable (Theorem 1.2) and quotient incomparable (Theorem 1.5) Banach spaces should be compared with the following general result of Rudin.

1.6. Theorem [42, Theorem 1.2]. Suppose $Y$ and $Z$ are subspaces of a Banach space $X$, and suppose that there is a bounded subset $\Phi \subset L(X)$ of operators mapping $X$ into $Y$ and $Z$ into $Z$, and such that to every $y \in Y$ and to every $\varepsilon>0$ there corresponds $A \in \Phi$ so that $\|y-A y\|<\varepsilon$. Then $Y+Z$ is closed.

Note that in every infinite-dimensional Banach space there exist closed subspaces with intersection $\{0\}$ and nonclosed sum [42, Proposition 4.8].

It is useful to introduce the following notion of projection incomparability, which is analogous to Definitions 1.1 and 1.4, but uses complemented subspaces.

1.7. Definition. The Banach spaces $X$ and $Y$ are said to be projection incomparable if there is no infinite-dimensional complemented subspace of $X$ isomorphic to a complemented subspace of $Y$. 
Obviously, the three above-defined concepts of structural incomparability are symmetric; that is, $X$ and $Y$ are subspace (quotient, projection) incomparable if and only if so are $Y$ and $X$. We emphasize this, because later we will find nonsymmetric incomparability concepts.

Operational incomparability. Now we give the definitions of some incomparability concepts defined in terms of the classes of compact, strictly singular, strictly cosingular and inessential operators.

Let $\operatorname{Co}(X, Y)$ denote the class of all compact operators acting from $X$ into $Y$. Several authors, for example [39] and [45], have studied the structure of the product of $X$ and $Y$ when any operator from $X$ to $Y$ is compact. In this way, they have implicitly considered the following relation of incomparability.

1.8. Definition. We say that $(X, Y) \in \operatorname{Co}$ when $L(X, Y)=\operatorname{Co}(X, Y)$.

We observe that the relation Co is not symmetric: it is well known that, for $1 \leq r<p<\infty$, we have $\left(\ell_{p}, \ell_{r}\right) \in$ Co, but $\left(\ell_{r}, \ell_{p}\right) \notin$ Co (see [28, p. 76]). This is the reason for using ordered pairs in the notation.

Now we define two relations similar to Co. Recall that the class $S S(X, Y)$ of strictly singular operators from $X$ to $Y$ is defined [37, 1.9.2] by

$$
S S(X, Y):=\left\{T \in L(X, Y): T J_{M} \text { injection } \Rightarrow \operatorname{dim}(M)<\infty\right\} .
$$

Similarly, the class $S C(X, Y)$ of strictly cosingular operators from $X$ into $Y$ is defined $[37,1.10 .2]$ by

$$
S C(X, Y):=\left\{T \in L(X, Y): Q_{U} T \text { surjection } \Rightarrow \operatorname{dim}(Y / U)<\infty\right\} .
$$

Strictly singular operators were introduced by Kato [25] in order to extend some perturbation results to semi-Fredholm operators, and strictly cosingular operators were introduced by Pełczyński in [35] and [36] in the study of $C(K)$ spaces and $L_{1}(\mu)$-spaces.

We have $\operatorname{Co}(X, Y) \subset S S(X, Y) \cap S C(X, Y)$, but $S S(X, Y)$ and $S C(X, Y)$ are not comparable [37, 1.11.9].

1.9. Definition. (1) We say that $(X, Y) \in S S$ when $L(X, Y)=S S(X, Y)$.

(2) We say that $(X, Y) \in S C$ when $L(X, Y)=S C(X, Y)$.

Recall that a Banach space $X$ has the Dunford-Pettis property if any weakly compact operator from $X$ into a space $Y$ is completely continuous; i.e., it takes weakly convergent sequences into convergent sequences. Moreover, $X$ has the reciprocal Dunford-Pettis property if any completely continuous operator from $X$ into a space $Y$ is weakly compact; and $X$ has the Schur property if weakly convergent sequences in $X$ are convergent.

Examples of Banach spaces with the Dunford-Pettis property are the spaces $C(K)$ of continuous functions in a compact set $K$, and the spaces $L_{1}(\mu)$ of integrable functions. Moreover, $C(K)$ and Banach spaces containing no copies of $\ell_{1}$ have the reciprocal Dunford-Pettis property, and for any set $\Gamma$, the space $\ell_{1}(\Gamma)$ 
has the Schur property. We refer to [5] for other examples of Banach spaces with these properties.

1.10. R e m ark. The relations $S S$ and $S C$ are not symmetric. Since $\ell_{\infty}$ contains a copy of $\ell_{2}$, we have $\left(\ell_{2}, \ell_{\infty}\right) \notin S S$. On the other hand, as $\ell_{2}$ is reflexive, any operator of $L\left(\ell_{\infty}, \ell_{2}\right)$ is weakly compact. Then, since $\ell_{\infty}$ has the Dunford-Pettis property, every operator of $L\left(\ell_{\infty}, \ell_{2}\right)$ is completely continuous [37, 1.6.1]. If there exists a non-strictly singular operator $T \in L\left(\ell_{\infty}, \ell_{2}\right)$, then we can find a subspace $M \subset \ell_{\infty}$ isomorphic to $\ell_{2}$ in which $T$ is an isomorphism. Since the canonical basis $\left(e_{n}\right)$ of $\ell_{2}$ is weakly null and $T$ is completely continuous, $\left(T e_{n}\right)$ is norm null, a contradiction.

Also, $\ell_{1}$ has a quotient isomorphic to $\ell_{2}$; hence $\left(\ell_{1}, \ell_{2}\right) \notin S C$. However, any operator from $\ell_{2}$ into $\ell_{1}$ is compact; thus $\left(\ell_{2}, \ell_{1}\right) \in S C$.

Finally, we consider the essential incomparability, introduced in [15]. Recall that $T \in L(X, Y)$ is said to be inessential [38] (see also [37, 4.3.5, 26.7.2]), denoted by $T \in \operatorname{In}(X, Y)$, if $I-S T$ is a Fredholm operator for all $S \in L(Y, X)$, where $I$ is the identity operator on $X$. This means that $I-S T$ has finite-dimensional kernel and closed finite-codimensional range. Note that the classes of compact, strictly singular and strictly cosingular operators are contained in the class of inessential operators [37, 26.7.3].

1.11. Definition [15, Definition 1]. The Banach spaces $X$ and $Y$ are said to be essentially incomparable, denoted by $(X, Y) \in \operatorname{In}$, if $L(X, Y)=\operatorname{In}(X, Y)$.

1.12. Proposition [15, Proposition 1]. The relation In is symmetric:

$$
(X, Y) \in \operatorname{In} \Leftrightarrow(Y, X) \in \operatorname{In} .
$$

One of the remarkable features of essential incomparability is the abundance of pairs of spaces satisfying this relation, as shown in the next result, where $H^{\infty}$ denotes the space of bounded analytic functions on the disc.

1.13. Theorem ([15, Theorem 1] and [1, Theorem 2.4]). The Banach spaces $X$ and $Y$ are essentially incomparable in the following cases:

(1) $X$ is reflexive and $Y$ has the Dunford-Pettis property.

(2) $X$ has the reciprocal Dunford-Pettis property and $Y$ has the Schur property.

(3) $X$ contains no copies of $\ell_{\infty}$ and $Y=\ell_{\infty}, H^{\infty}$ or $C(K)$ with $K \sigma$-stonian.

(4) $X$ contains no copies of $c_{0}$ and $Y=C(K)$.

(5) $X$ contains no complemented copies of $c_{0}$ and $Y=C[0,1]$.

(6) $X$ contains no complemented copies of $\ell_{1}$ and $Y=L_{1}(\mu)$.

(7) $X$ contains no complemented copies of $\ell_{p}, 1<p<\infty$, and $Y=L_{p}[0,1]$, or $\ell_{p}$.

Moreover, essential incomparability admits the following structural characterizations:

1.14. TheOREM [15, Theorem 2]. The following assertions are equivalent: 
(1) The Banach spaces $X$ and $Y$ are essentially incomparable.

(2) For any Banach space $Z$ and complemented subspaces $X_{0}$ and $Y_{0}$ of $Z$, isomorphic to $X$ and $Y$ respectively, $X_{0} \cap Y_{0}$ is finite-dimensional.

(3) For any Banach space $Z$ and complemented subspaces $X_{0}$ and $Y_{0}$ of $Z$, isomorphic to $X$ and $Y$ respectively, $X_{0}+Y_{0}$ is complemented.

(4) For any Banach space $Z$ and complemented subspaces $X_{0}$ and $Y_{0}$ of $Z$, isomorphic to $X$ and $Y$ respectively, $X_{0}+Y_{0}$ is closed.

Relations between the incomparability concepts. The above-defined seven concepts of incomparability are related as we show in the following result, which can be derived from the definitions and the inclusion relations between the classes of operators implied.

1.15. Proposition. (1) If $X$ and $Y$ are subspace incomparable, then $(X, Y) \in$ $S S$ and $(Y, X) \in S S$. $S C$.

(2) If $X$ and $Y$ are quotient incomparable, then $(X, Y) \in S C$ and $(Y, X) \in$

(3) If $(X, Y) \in$ Co, then $(X, Y) \in S S$ and $(X, Y) \in S C$.

(4) If $(X, Y) \in S S$ or $(X, Y) \in S C$, then $X$ and $Y$ are essentially incomparable.

(5) If $X$ and $Y$ are essentially incomparable, then they are projection incomparable.

We summarize the above relations in the following diagram, where $S I(Q I, P I)$ means subspace (quotient, projection) incomparable, and $\rightarrow$ means "implies".

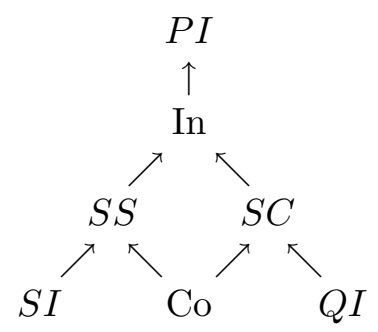

For the six concepts of incomparability $S I$, Co, $Q I, S S, S C$ and In there are no additional implications to those showed in the above diagram, as shown by the following examples.

1.16. EXAMPLE. The sequence spaces $\ell_{1}$ and $\ell_{2}$ are subspace incomparable, hence $\left(\ell_{1}, \ell_{2}\right) \in S S$. But $\left(\ell_{1}, \ell_{2}\right) \notin S C$, since $\ell_{1}$ has a quotient isomorphic to $\ell_{2}$; hence $\left(\ell_{1}, \ell_{2}\right) \notin$ Co also.

1.17. Example. The spaces $c_{0}$ and $\ell_{\infty}$ are quotient incomparable [18], hence $\left(c_{0}, \ell_{\infty}\right) \in S C$. But $\left(c_{0}, \ell_{\infty}\right) \notin S S$, since $c_{0}$ is isomorphic to a subspace of $\ell_{\infty}$. Hence they are not subspace incomparable and $\left(c_{0}, \ell_{\infty}\right) \notin$ Co.

1.18. EXAMPLE. Since $\ell_{1}$ is a separable Banach space, every operator of $L\left(\ell_{\infty}, \ell_{1}\right)$ is weakly compact [28]. Moreover, $\ell_{1}$ has the Schur property. Hence 
$\left(\ell_{\infty}, \ell_{1}\right) \in$ Co. However, $\ell_{\infty}$ contains a subspace isomorphic to $\ell_{1}$, hence $\ell_{\infty}$ and $\ell_{1}$ are not subspace incomparable. Moreover, $\ell_{2}$ is isomorphic to a quotient space of $\ell_{\infty}\left[28\right.$, p. 111] and to a quotient space of $\ell_{1}$; hence $\ell_{1}$ and $\ell_{\infty}$ are not quotient incomparable.

1.19. ExAmPLE. The spaces $\ell_{2}(C[0,1])$ and $L_{1}[0,1]$ are essentially incomparable, since $\ell_{2}(C[0,1])$ contains no complemented copies of $\ell_{1}$ (see [15, Theorem 1]). However, $\ell_{2}$ is isomorphic to a complemented subspace of $\ell_{2}(C[0,1])$ and to an (uncomplemented) subspace of $L_{1}[0,1]$; thus the operator

$$
T: \ell_{2}(C[0,1])=\ell_{2} \oplus M \rightarrow \ell_{2} \subset L_{1}[0,1],
$$

defined by $T(x+y)=x, x \in \ell_{2}$ and $y \in M$, is not strictly singular; hence $\left(\ell_{2}(C[0,1]), L_{1}[0,1]\right) \notin S S$. Analogously, since $\ell_{1}$ is isomorphic to an (uncomplemented) subspace of $\ell_{2}(C[0,1])$ and to a complemented subspace of $L_{1}[0,1]$, we have $\left(L_{1}[0,1], \ell_{2}(C[0,1])\right) \notin S S$. Moreover, $\ell_{2}(C[0,1])$ is isomorphic to a quotient space of $\ell_{1}$. Hence $\left(L_{1}[0,1], \ell_{2}(C[0,1])\right) \notin S C$.

It is not known, in the general case, if the converse of the remaining implication is true.

1.20. Open PROBlem [15]. Are the concepts of projection incomparability and essential incomparability equivalent?

In [1] a partial answer to this problem is given, showing that the answer is positive when one of the spaces has "enough" projections. In order to make precise the result, recall that $X$ is said to be subprojective if for each infinitedimensional subspace $N$ of $X$ there exists an infinite-dimensional subspace $M \subset$ $N$ which is complemented in $X$. Also, $X$ is said to be superprojective if for any infinite-codimensional subspace $N$ of $X$, there exists an infinite-codimensional subspace $M \supset N$ which is complemented in $X$. The spaces $\ell_{p}$ are subprojective and superprojective for $1<p<\infty$, and the spaces $L_{p}[0,1]$ are subprojective for $2<p<\infty$ and superprojective for $1<p<2$ [43]. For other examples we refer to $[1]$.

1.21. THEOREM [1]. Assume $X$ (or $Y$ ) is subprojective or superprojective. Then the following equivalence holds: $X$ and $Y$ are essentially incomparable if and only if they are projection incomparable.

In the next result we collect the duality properties of the concepts of incomparability.

1.22. TheOREM [15], [18]. (1) If $X^{*}$ and $Y^{*}$ are subspace, quotient or projection incomparable, then $X$ and $Y$ are quotient, subspace or projection incomparable, respectively.

(2) If $\left(X^{*}, Y^{*}\right) \in \mathrm{Co}, S S, S C$, In, then $(Y, X) \in \mathrm{Co}, S C, S S$, In, respectively.

1.23. Remark. The converse implications in the above result fail in a very strong sense: 
Bourgain and Delbaen [6] constructed a hereditarily reflexive Banach space $Z$ whose dual $Z^{*}$ is isomorphic to $\ell_{1}$. It is not difficult to see that $c_{0}$ and $Z$ are subspace incomparable and $\left(c_{0}, Z\right) \in$ Co. However, the dual spaces are both isomorphic to $\ell_{1}$; hence $\left(c_{0}^{*}, Z^{*}\right),\left(Z^{*}, c_{0}^{*}\right) \notin \mathrm{In}$.

Also, as we have mentioned before, $c_{0}$ and $\ell_{\infty}$ are quotient incomparable and $\left(c_{0}, \ell_{\infty}\right) \in$ Co. However, $c_{0}^{*}$ is isomorphic to a complemented subspace of $\ell_{\infty}^{*}$, hence $\left(c_{0}^{*}, \ell_{\infty}^{*}\right),\left(\ell_{\infty}^{*}, c_{0}^{*}\right) \notin \mathrm{In}$.

2. Applications to Banach space theory. Here we collect the main applications of the incomparability concepts introduced in the first section.

Complemented subspaces of a direct sum. The first application is the description of the complemented subspaces of a product of incomparable spaces.

2.1. THEOREM [15, Theorem 2]. If $X$ and $Y$ are essentially incomparable, and $Z \subset X \oplus Y$ is a complemented subspace, then $Z$ is isomorphic to $X_{0} \oplus Y_{0}$, with $X_{0}$ and $Y_{0}$ complemented subspaces of $X$ and $Y$, respectively.

In 1976, Edelstein and Wojtaszczyk [13, Theorem 3.5] proved the above result assuming $X$ and $Y$ to be totally incomparable, and in 1978 Wojtaszczyk [44, Theorem 1.1] proved it under the weaker assumption $(X, Y) \in S S$. Also Prada [39, Theorem 3] proved this result in 1984 for $X$ and $Y$ Fréchet spaces such that $(X, Y) \in \mathrm{Co}$

2.2. Proposition [15, Lemma 1]. If $P$ and $Q$ are projections in a Banach space $X$ and $P Q \in \operatorname{In}$ (in particular, if the ranges $R(P)$ and $R(Q)$ are essentially incomparable), then $R(P) \cap R(Q)$ is finite-dimensional, and $R(P)+R(Q)$ is complemented in $X$.

This result was proved by Lavergne [27] for $P Q$ strictly singular.

Other results about complemented subspaces of nonatomic Banach lattices were proved by Casazza, Kalton and Tzafriri [7]. For the concepts of nonatomic Banach lattice and type and cotype of a Banach space, we refer to the book of Lindenstrauss and Tzafriri [29].

2.3. Theorem [7, Theorem 2.1]. Let $Z$ be a nonatomic Banach lattice with nontrivial cotype, and suppose $Z \cong X \oplus Y$, where $X$ and $Y$ are subspace incomparable. Then either $X$ or $Y$ is finite-dimensional.

2.4. Theorem [7, Theorem 2.2]. Let $Z$ be a nonatomic Banach lattice with nontrivial cotype, and suppose $Z \cong X \oplus Y$, with $(X, Y) \in S S$. Then each of the following conditions implies $X$ is finite-dimensional:

(1) $X$ contains no copies of $\ell_{2}$.

(2) $Z$ has nontrivial type and $X$ contains no complemented copies of $\ell_{2}$.

(3) $Z$ has nontrivial type and $Y$ has an infinite-dimensional complemented subspace with an unconditional basis. 
Unconditional bases of a direct sum. Another application of incomparability is the decomposition of an unconditional basis of the direct sum of two incomparable Banach spaces into unconditional bases of the factors.

2.5. Theorem [44, Theorem 2.1]. If $(X, Y) \in \mathrm{Co}$, then every unconditional basis of $X \oplus Y$ splits into two disjoint parts equivalent to (unconditional) bases of $X$ and $Y$, respectively.

In 1987, Casazza, Kalton and Tzafriri [7] gave another sufficient condition for the result, and posed an open problem.

2.6. TheOREM [7, Theorem 1.5]. If $X$ and $Y$ are subspace incomparable and have each an unconditional basis, then every unconditional basis of $X \oplus Y$ splits into two disjoint parts which are respectively equivalent to (unconditional) bases of $X$ and $Y$.

2.7. Open problem [7]. Assume $(X, Y) \in S S$. Does every unconditional basis of $X \oplus Y$ split into two disjoint parts equivalent to unconditional bases of $X$ and $Y$, respectively?

Structure of tensor products. The structure of the injective and surjective tensor products of two Banach spaces, as well as the structure of spaces of operators, is not well understood. Some results have been found describing the structure of the product in terms of the structure of the factors, when the spaces satisfy a suitable incomparability condition. As a sample, we give here a classical result and a more recent one.

2.8. Theorem [10, Theorem VIII.4.4]. Assume $X$ and $Y$ are reflexive, and one of them has the approximation property. Then $L(X, Y)$ is reflexive if and only if $(X, Y) \in$ Co.

2.9. Theorem [14, Theorem 3]. Assume $\left(X, Y^{*}\right) \in$ Co. If $X$ and $Y$ contain no copies of $\ell_{1}$, then $X \otimes_{\pi} Y$ does not contain copies of $\ell_{1}$.

Spaces whose group of invertible operators is not connected. Several authors have studied the homotopy type of the group of invertible operators in some classical Banach spaces. For example, in 1965 Kuiper [26] proved the contractibility of the group of invertible operators in a Hilbert space, and in 1967 Neubauer [34] obtained the same result for the spaces $\ell_{p}, 1<p<\infty$, and $c_{0}$. The first example of a Banach space with disconnected group of invertible operators is due to Douady [11], in 1965. The survey paper of Mityagin [33] gives additional information up to 1970 .

Using essentially incomparable spaces, it is possible to construct examples of Banach spaces whose group of invertible operators is not connected. Note that a hyperplane is a 1-codimensional (closed) subspace, and it was shown by Gowers and Maurey [20], in 1992, that there exist infinite-dimensional Banach spaces not isomorphic to their hyperplanes, solving a long standing open problem. 
2.10. THEOREM [15, Theorem 3]. If $X$ and $Y$ are essentially incomparable, and both isomorphic to their respective hyperplanes, then the group of all invertible operators in $X \oplus Y$ is not connected.

Classes of incomparability. The concepts of subspace incomparability and quotient incomparability have allowed giving procedures of construction of space ideals with the three-space property.

2.11. Definition [3, Definition 2.4]. For a nonempty class $G$ of Banach spaces, we define

(1) $G^{\mathrm{s}}:=\{X: \forall Y \in G, X$ and $Y$ are subspace incomparable $\}$,

(2) $G^{\mathrm{q}}:=\{X: \forall Y \in G, X$ and $Y$ are quotient incomparable $\}$.

Simple properties of the classes $G^{\mathrm{s}}$ and $G^{\mathrm{q}}$ are the following:

$$
G \subset G^{\mathrm{ss}} \cap G^{\mathrm{qq}}, \quad G^{\mathrm{s}}=G^{\mathrm{sss}} \quad \text { and } \quad G^{\mathrm{q}}=G^{\mathrm{qqq}} .
$$

Moreover, $G^{\mathrm{s}}$ and $G^{\mathrm{q}}$ are well-behaved space ideals. Recall that a space ideal $A$ is a class of Banach spaces which includes the finite-dimensional Banach spaces, and is stable under isomorphisms, finite products and complemented subspaces. A space ideal $A$ is injective if every subspace of $X \in A$ belongs to $A$, it is surjective if every quotient of $X \in A$ belongs to $A$, and it has the three-space property when given a subspace $M$ of $X$ such that $M, X / M \in A$, we have $X \in A$. We refer to the book of Pietsch [37] for an account of the general theory of space ideals, and to [19] for a survey about space ideals with the three-space property.

2.12. TheOREM [3, Theorem 2.5]. If $G$ is a nonempty class of Banach spaces, then

(1) $G^{\mathrm{s}}$ is a three-space injective ideal,

(2) $G^{\mathrm{q}}$ is a three-space surjective ideal.

In this way, we have a procedure that applied, for example, to the class $R$ of all reflexive Banach spaces, gives four space ideals with the three-space property: $R^{\mathrm{s}}$ (spaces with no reflexive subspaces), $R^{\mathrm{ss}}$ (somewhat reflexive spaces), $R^{\mathrm{q}}$ and $R^{\mathrm{qq}}$.

A family of concrete examples obtained by means of this procedure can be shown, using the concepts of minimal and cominimal space. Recall that a Banach space $X$ is said to be minimal if every infinite-dimensional subspace of $X$ contains a copy of $X$, and it is said to be cominimal if every infinite-dimensional quotient of $X$ has a quotient isomorphic to $X$. The spaces $c_{0}, \ell_{p}(1 \leq p<\infty)$ and $T^{*}$ (Tsirelson space) are minimal; $c_{0}$ and the dual spaces of reflexive minimal spaces are cominimal.

2.13. Example [3]. If $X$ is minimal, then

$$
\{X\}^{\mathrm{s}}=\{Y: Y \text { contains no copies of } X\} .
$$


If $X$ is cominimal, then

$$
\{X\}^{\mathrm{q}}=\{Y: Y \text { has no quotient isomorphic to } X\} .
$$

2.14. OPEN PROBLEM. Is it possible to obtain procedures for construction of space ideals with the three-space property, using other incomparability concepts?

Operational quantities. Space ideals $A$ satisfying the conditions $A=A^{\text {ss }}$ and $A=A^{\text {qq }}$ have been applied to define and study operator ideals. Note that these conditions are satisfied by the space ideal $F$ of finite-dimensional Banach spaces. Using certain operational quantities associated with a space ideal $A$, it is possible to define classes of operators which generalize the classes $S S$ and $S C$ of strictly singular and strictly cosingular operators, and the classes $S F_{+}$and $S F_{-}$of semiFredholm operators. We refer to [32] for a complete account of this subject.

Let $A$ denote a space ideal different from the class of all Banach spaces. We shall need the auxiliary classes $A_{\mathrm{s}}$ and $A_{\mathrm{q}}$ given by

$$
\begin{aligned}
& A_{\mathrm{s}}=\{X: M \text { subspace of } X \Rightarrow M \in A\}, \\
& A_{\mathrm{q}}=\{X: U \text { subspace of } X \Rightarrow X / U \in A\} .
\end{aligned}
$$

Using these classes it is possible to derive some quantities from the norm, which we denote by $n(T):=\|T\|$.

2.15. Definition [16, Definition 5]. Let $A$ be a space ideal different from the class of all Banach spaces, and $T \in L(X, Y)$. We define the following operational quantities:

1. If $X \notin A_{\mathrm{s}}$, then

(a) $\operatorname{in}_{A}(T):=\inf \left\{n\left(T J_{M}\right): M \notin A\right\}$,

(b) $\sin _{A}(T):=\sup \left\{\operatorname{in}_{A}\left(T J_{M}\right): M \notin A\right\}$.

2. If $Y \notin A_{\mathrm{q}}$, then

(a) $\operatorname{in}_{A}^{\prime}(T):=\inf \left\{n\left(Q_{U} T\right): Y / U \notin A\right\}$,

(b) $\sin _{A}^{\prime}(T):=\sup \left\{\operatorname{in}_{A}^{\prime}\left(Q_{U} T\right): Y / U \notin A\right\}$.

Now we introduce the classes of operators defined in terms of these operational quantities.

2.16. Definition [16, Definition 8 and Definition 13]. For any Banach spaces $X$ and $Y$, we define the following classes of operators:

(1) $S A_{+}(X, Y):= \begin{cases}L(X, Y) & \text { if } X \in A_{\mathrm{s}}, \\ \left\{T \in L(X, Y): \operatorname{in}_{A}(T)>0\right\} & \text { if } X \notin A_{\mathrm{s}},\end{cases}$

(2) $A S S(X, Y):= \begin{cases}L(X, Y) & \text { if } X \in A_{\mathrm{S}}, \\ \left\{T \in L(X, Y): \sin _{A}(T)=0\right\} & \text { if } X \notin A_{\mathrm{S}},\end{cases}$

(3) $S A_{-}(X, Y):= \begin{cases}L(X, Y) & \text { if } Y \in A_{\mathrm{q}}, \\ \left\{T \in L(X, Y): \operatorname{in}_{A}^{\prime}(T)>0\right\} & \text { if } Y \notin A_{\mathrm{q}},\end{cases}$

(4) $A S C(X, Y):= \begin{cases}L(X, Y) & \text { if } Y \in A_{\mathrm{q}} \\ \left\{T \in L(X, Y): \sin _{A}^{\prime}(T)=0\right\} & \text { if } Y \notin A_{\mathrm{q}} \text {. }\end{cases}$ 
If $A=F$, we obtain $S A_{+}=S F_{+}$, the class of upper semi-Fredholm operators (operators that have finite-dimensional kernel and closed range), and $S A_{-}=S F_{-}$, the class of lower semi-Fredholm operators (operators that have finite-codimensional range). Moreover, $S F_{+} \subset S A_{+}$and $S F_{-} \subset S A_{-}$, and the classes $S A_{+}(X, Y)$ and $S A_{-}(X, Y)$ are open in $L(X, Y)$. Also, the classes $S A_{+}$ and $S A_{-}$are empty in some cases.

2.17. Proposition [16, Proposition 9]. (1) If $A=A^{\mathrm{ss}}, X \notin A_{\mathrm{s}}$ and $Y \in A_{\mathrm{s}}$, then $S A_{+}(X, Y)=\emptyset$.

(2) If $A=A^{\mathrm{qq}}, X \in A_{\mathrm{q}}$ and $Y \notin A_{\mathrm{q}}$, then $S A_{-}(X, Y)=\emptyset$.

If $A=F$, we have $F S S=S S$ and $F S C=S C$. Also, for any $A$, the classes $A S S$ and $A S C$ are closed in $L(X, Y)$.

There are some unsolved questions about the above classes. Recall that an operator ideal is a class of linear continuous operators which contains the finite rank operators, is closed under addition and is stable under composition with continuous operators.

2.18. Open problem. Are $A S S$ and $A S C$ operator ideals?

A partial answer to this problem has been obtained using the notions of subspace incomparability and quotient incomparability.

2.19. Theorem [16, Theorem 14]. (1) If $A=A^{\mathrm{ss}}$, then $A S S$ is an operator ideal and

$$
A S S(X, Y)=\left\{T \in L(X, Y): T J_{M} \text { injection } \Rightarrow M \in A\right\} .
$$

(2) If $A=A^{\mathrm{qq}}$, then $A S C$ is an operator ideal and

$$
A S C(X, Y)=\left\{T \in L(X, Y): Q_{U} T \text { surjection } \Rightarrow Y / U \in A\right\} .
$$

Taking the equalities of the above theorem as definitions, the classes $A S S$ and $A S C$ have been studied in [4]. We do not know if $S S \subset A S S$ and $S C \subset A S C$ for every space ideal $A$, although the class of compact operators Co is contained in both classes $A S S$ and $A S C$ [16, Remark 16].

2.20. Open PROBlem. Is it true that $S S \subset A S S$ and $S C \subset A S C$ ?

In the case $T \in L(X, X)$, it would be interesting to study the asymptotic behaviour of the quantities $\sin _{A}\left(T^{n}\right)^{1 / n}$, and analogously for $\sin _{A}^{\prime}(T), \operatorname{in}_{A}(T)$ and $\operatorname{in}_{A}^{\prime}(T)$. In the classical case ( $A$ the finite-dimensional spaces) these limits exist, and coincide with the essential spectral radius for the first two quantities, and with the upper semi-Fredholm radius and the lower semi-Fredholm radius for the remaining two. We refer to [46] for details.

2.21. Open problem. Given $T \in L(X, X)$, what is the limit of $q\left(T^{n}\right)^{1 / n}$, for $q$ one of the quantities $\sin _{A}(T)$ and $\sin _{A}^{\prime}(T), \operatorname{in}_{A}(T)$ or $\operatorname{in}_{A}^{\prime}(T)$ ? 
Finally, we show that $A S S, A S C, S A_{+}$and $S A_{-}$behave like the classes of Fredholm theory, in the sense that $S A_{+}$and $S A_{-}$are stable under perturbation by elements of $A S S$ and $A S C$, respectively.

2.22. Theorem [16, Theorem 17]. (1) If $T \in S A_{+}$and $K \in A S S$, then $T+K$ $\in S A_{+}$.

(2) If $T \in S A_{-}$and $K \in A S C$, then $T+K \in S A_{-}$.

In [17] other classes of operators, associated with operational quantities defined using the injection modulus and the surjection modulus instead of the norm, are studied.

\section{References}

[1] P. Aiena and M. González, Essentially incomparable Banach spaces and Fredholm theory, Proc. Roy. Irish Acad. 93A (1993), 49-59.

[2] T. Alvarez, M. González and V. M. Onieva, A note on three-space Banach space ideals, Arch. Math. (Basel) 46 (1986), 169-170.

[3] —, - - - Totally incomparable Banach spaces and three-space ideals, Math. Nachr. 131 (1987), 83-88.

[4] —, - - - Characterizing two classes of operator ideals, in: Contribuciones Matemáticas. Homenaje Prof. Antonio Plans, Univ. Zaragoza, Zaragoza, 1990, 7-21.

[5] J. Bourgain, New Classes of $\mathcal{L}^{p}$-Spaces, Lecture Notes in Math. 889, Springer, Berlin, 1981.

[6] J. Bourgain and F. Delbaen, A class of special $\mathcal{L}^{\infty}$-spaces, Acta Math. 145 (1980), $155-176$.

[7] P. G. Casazza, N. J. Kalton and L. Tzafriri, Decompositions of Banach lattices into direct sums, Trans. Amer. Math. Soc. 304 (1987), 771-800.

[8] J. C. Díaz, An example of Fréchet space, not Montel, without infinite dimensional normable subspaces, Proc. Amer. Math. Soc. 96 (1986), 721.

[9] J. Diestel and R. H. Lohman, Applications of mapping theorems to Schwartz spaces and projections, Michigan Math. J. 20 (1973), 39-44.

[10] J. Diestel and J. J. Uhl, Jr., Vector Measures, Math. Surveys 15, Amer. Math. Soc., 1977.

[11] A. Douady, Un espace de Banach dont le groupe linéaire n'est pas connexe, Indag. Math. 27 (1965), 787-789.

[12] L. Drewnowski, On minimally subspace-comparable F-spaces, J. Funct. Anal. 26 (1977), $39-44$.

[13] I. S. Edelstein and P. Wojtaszczyk, On projections and unconditional bases in direct sums of Banach spaces, Studia Math. 56 (1976), 263-276.

[14] G. Emmanuele, Banach spaces in which Dunford-Pettis sets are relatively compact, Arch. Math. (Basel) 58 (1992), 477-485.

[15] M. González, On essentially incomparable Banach spaces, Math. Z., to appear.

[16] M. González and A. Martinón, Operational quantities derived from the norm and generalized Fredholm theory, Comment. Math. Univ. Carolin. 32 (1991), 645-657.

[17] —, - Fredholm theory and space ideals, Boll. Un. Mat. Ital. 7-B (1993), 473-488.

[18] M. González and V. M. Onieva, On incomparability of Banach spaces, Math. Z. 112 (1986), 581-585.

[19] —, - El problema de los tres espacios, in: Homenaje al Prof. Dr. Nácere Hayek Calil, Univ. La Laguna, La Laguna, 1990, 155-162. 
[20] W. T. Gowers and B. Maurey, The unconditional basic sequence problem, J. Amer. Math. Soc. 6 (1993), 851-874.

[21] V. I. Gurariŭ, On openings and inclinations of subspaces of Banach spaces, Teor. Funktsiu Funktsional. Anal. i Prilozhen. 1 (1965), 194-204 (in Russian).

[22] S. Heinrich, On the relation between linear $n$-widths and approximation numbers, J. Approx. Theory 58 (1989), 315-333.

[23] N. J. Kalton, Quotients of F-spaces, Glasgow Math. J. 19 (1978), 103-108.

[24] N. J. Kalton, B. Turett and J. J. U hl, Jr., Basically scattered vector measures, Indiana Univ. Math. J. 28 (1979), 803-816.

[25] T. Kato, Perturbation theory for nullity, deficiency and other quantities of linear operators, J. Analyse Math. 6 (1958), 261-322.

[26] N. H. Kuiper, The homotopy type of the unitary group of Hilbert space, Topology 3 (1965), 19-30.

[27] A. Lavergne, Remark on sums of complemented subspaces, Colloq. Math. 41 (1979), 103-104.

[28] J. Lindenstrauss and L. Tzafriri, Classical Banach Spaces I. Sequence Spaces, Springer, Berlin, 1977.

[29] -, -, Classical Banach Spaces II. Function Spaces, Springer, Berlin, 1977.

[30] R. H. Lohman, An example concerning normed subspaces of locally convex spaces, Proc. Amer. Math. Soc. 41 (1973), 245-246.

[31] A. Martinón, Cantidades operacionales en teoría de Fredholm, Tesis Doctoral, Univ. La Laguna, 1989.

[32] - Operational quantities and classes of operators, in: Aportaciones matemáticas en memoria del Prof. V. M. Onieva, Univ. Cantabria, Santander, 1991, 227-236.

[33] B. S. Mityagin, The homotopy structure of the linear group of a Banach space, Russian Math. Surveys 25 (1970), 59-103.

[34] G. Neubauer, Der Homotopietyp der Automorphismengruppe in den Räumen $l_{p}$ und $c_{0}$, Math. Ann. 174 (1967), 33-40.

[35] A. Pełczyński, On strictly singular and strictly cosingular operators. I. Strictly singular and strictly cosingular operators in $C(S)$-spaces, Bull. Acad. Polon. Sci. Sér. Sci. Math. Astronom. Phys. 13 (1965), 31-36.

[36] - On strictly singular and strictly cosingular operators. II. Strictly singular and strictly cosingular operators in $L(\nu)$-spaces, ibid., 37-41.

[37] A. Pietsch, Operator Ideals, North-Holland, Amsterdam, 1980.

[38] - Inessential operators in Banach spaces, Integral Equations Operator Theory 1 (1978), 589-591.

[39] J. Prada, On idempotent operators on Fréchet spaces, Arch. Math. (Basel) 43 (1984), 179-182.

[40] H. P. Rosenthal, On totally incomparable Banach spaces, J. Funct. Anal. 4 (1969), 167175.

[41] -, On quasi-complemented subspaces of Banach spaces, with an appendix on compactness of operators from $L^{p}(\mu)$ to $L^{r}(\nu)$, ibid., 176-214.

[42] W. Rudin, Spaces of type $H^{\infty}+C$, Ann. Inst. Fourier (Grenoble) 25 (1) (1975), 99-125.

[43] R. J. Whitley, Strictly singular operators and their conjugates, Trans. Amer. Math. Soc. 113 (1964), 252-261.

[44] P. Wojtaszczyk, On projections and unconditional bases in direct sums of Banach spaces II, Studia Math. 62 (1978), 193-201.

[45] V. P. Zahariuta, On the isomorphism of cartesian products of locally convex spaces, ibid. 46 (1973), 201-221.

[46] J. Zemánek, Geometric characteristics of semi-Fredholm operators and their asymptotic behaviour, ibid. 80 (1984), 219-234. 